\title{
SIMULATION ON AN OPTIMAL COMBUSTION CONTROL STRATEGY FOR 3-D TEMPERATURE DISTRIBUTIONS IN PC-FIRED UTILITY BOILER FURNACES
}

\author{
Xi-Fen Wang *, Da-Wei Wang *, Huai-Chun Zhou *, \\ Jin He**, Zheng-Lin Pei**, Chuan-Xin Lu** \\ * State Key Laboratory of Coal Combustion, Huazhong University of Science and Technology, \\ Wuhan 430074, China, ${ }^{* *}$ Wuhan Steel Electricity Co. Ltd., Wuhan 430082, China
}

\begin{abstract}
The 3-D temperature distribution in a utility boiler furnace is essential for the safe, economic and clean operation of PC-fired furnaces with multi-burner system. In the past, due to the lack of applicable techniques to monitor the 3-D temperature distributions in furnaces, it is difficult to improve the control quality for the combustion processes. The authors is now establishing a technique to visualize 3-D temperature distributions in PC-fired furnace, which makes it possible to study a new combustion control strategy. In this strategy, the whole height of the furnace is divided into several parts, and the average temperatures, the bias magnitudes of the flame centers in the two axis of the horizontal plane in every part is extracted from the visualization results of the 3-D temperature distributions. A linear model is set up to relate the features of the temperature distributions with the input of the combustion processes, such as the flow rates of fuel and air fed into the furnaces through all the burners. The genetic algorithm is adopted to find the optimal combination of the whole input parameters which ensures to form an optimal 3-D temperature field in the furnace desired for the operation of boiler. Simulation results show that the strategy can soon find the factors causing the temperature distribution apart from the optimal state and give correct adjusting suggestions.
\end{abstract}

Keywords: Multi-burner furnaces, PC-fired boilers, Combustion control, Temperature distributions, Genetic algorithm

\section{INTRODUCTION}

Real-time, on-line optimal control techniques are essential for the safe, economy and clean operation of large amount of fossil-fuel-fired, utility and industrial boilers in the world. At present, the input variables of the combustion system in a pulverized-coal-fired boiler, including the fuels, primary airs, secondary airs and tertiary airs of all burners, always be controlled via the bias of the main steam pressure with respect to the preset value, the oxygen and/or CO contents in the flue gas [1]. Recently, the $N O_{x}$ emissions in the flue exhaust were also proposed to adjust the combustion processes [2-4]. In fact, whether the contents of gas components in flue gases [1-4] or the steam parameters [5] are used as input parameters, the delay is too long compared with the time scale of the combustion reactions. So, it will be a good choice to monitor simultaneously the temperature distributions [6-8] and the intermediate products [11] inside furnaces to accelerate the responding speeds of combustion control systems.

In this paper, a furnace combustion control strategy using a group of new characteristic parameters featuring the 3-D temperature distributions inside the furnace as intermediate controlled parameters, which can avoid the deficiency of long delay in conventional combustion control systems and organize an optimal 3-D temperature distribution inside the furnace, is propose and studied by simulation. The control strategy adopted Genetic Algorithm to search for the optimal control signals. The control strategy showed good capability to continuously adjust the flow rates of the fuels, the primary airs and the secondary airs of each burner and keep the boiler combustion under optimal temperature distribution conditions. The details are described as below.

\section{THE MODEL OF 3-D TEMPERATURE DISTRIBUTION IN FURNACE}

The 3-D temperature distribution in a furnace is discretized at $m$ planes along the height of the furnace, as shown in Fig. 1. The fourth power of the average temperature values, $T_{i}^{4}$, indicating the emission energy level of the flame, and the rectangular coordinates, $\left(x_{i}, y_{i}\right)$, of the migration of the flame centers with respect to the geometric centers, $(0,0)$, in the horizontal planes, which make up of the distribution feature of the 3-D flame in the furnace, can present the 3-D characteristics of the whole combustion process inside the furnace, and can be obtained from the 3-D temperature distributions of 


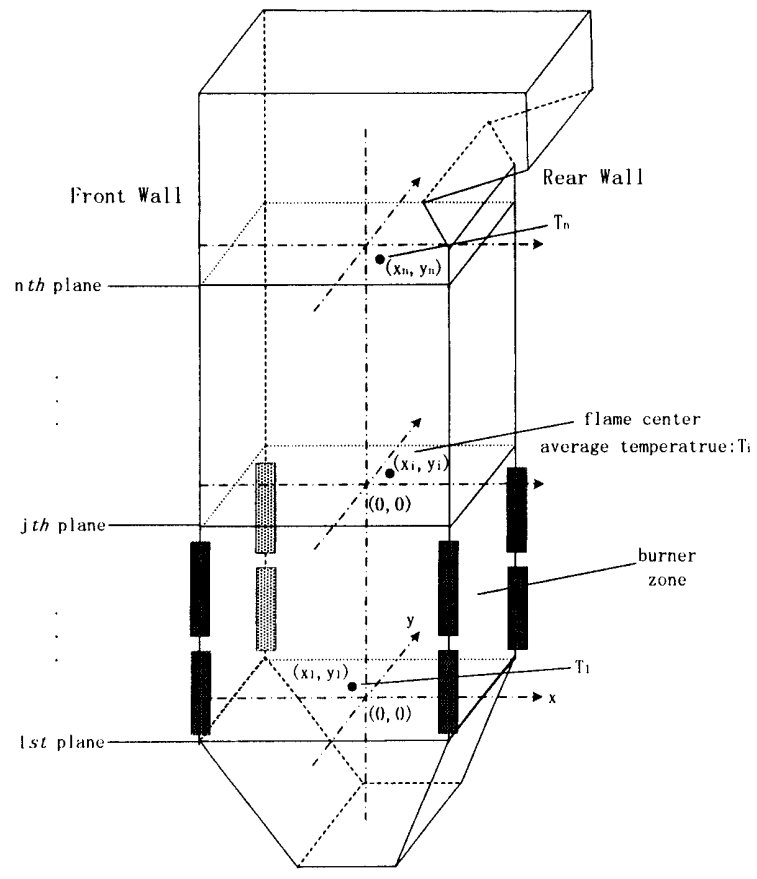

Fig. 1 The representation of the 3-D temperature distribution in a tangentially firing furnace.

combustion reconstructed from flame image processing techniques [6]. So, the output parameter vector of the combustion control objective is represented as follows,

$$
S=\left(T_{1}^{4}, x_{1}, y_{1}, T_{2}^{4}, x_{2}, y_{2}, \cdots, T_{m}{ }^{4}, x_{m p} y_{m}\right)^{T} \text {. }
$$

The input parameter vector, which is composed of every flow rate of the fuels, $F_{j}$, primary and secondary airs, $A 1_{j}$ and $A 2_{j}$, respectively, of all burners, and the tertiary air, $A 3$, is written as

$$
\begin{aligned}
U & =\left(U_{1}, U_{2}, \cdots, U_{n}, U_{n+1}\right)^{T} \\
& =\left(F_{1}, A 1_{1}, A 2_{1}, F_{2}, A 1_{2}, A 2_{2}, \cdots, F_{n}, A 1_{n}, A 2_{n}, A 3\right)^{T}
\end{aligned}
$$

where, $n$ refers to the number of the burners. The mathematic model relating the 3-D temperature distribution of flames, $S$, with the input parameter vector, $U$, in furnace is simplified as,

$$
S=D U
$$

where $D$ is a coefficient matrix with dimensions of $3 m \times(3 n+1)$.

The input and output parameter vectors are further reorganized as

$$
\begin{aligned}
S= & \left(T_{1}{ }^{4}, T_{2}{ }^{4}, \cdots, T_{m}{ }^{4}, x_{1}, x_{2}, \cdots, x_{m}, y_{1}, y_{2}, \cdots, y_{m}\right)^{T}, \\
& =\left(T^{4}, X, Y\right)^{T}
\end{aligned}
$$

and

$$
\begin{aligned}
U & =\left(F_{1}, F_{2}, \cdots, F_{n}, A 1_{1}, A 1_{2}, \cdots, A 1_{n}, A 2_{1}, A 2_{2}, \cdots, A 2_{n}, A 3\right)^{T}, \\
& =(F, A 1, A 2, A 3)^{T}
\end{aligned}
$$

respectively. Then the coefficient matrix $D$ is revised as

$$
D=\left[\begin{array}{cccc}
D_{T F} & D_{T A 1} & D_{T A 2} & D_{T A 3} \\
D_{X F} & D_{X A 1} & D_{X A 2} & D_{X A 3} \\
D_{Y F} & D_{Y A 1} & D_{Y A 2} & D_{Y A 3}
\end{array}\right],
$$

and the mathematic model, Eq. (4), is changed to the following form

$$
\left\{\begin{array}{l}
T^{4}=D_{T F} F+D_{T A 1} A 1+D_{T A 2} A 2+D_{T A 3} A 3 \\
X=D_{X F} F+D_{X A 1} A 1+D_{X A 2} A 2+D_{X A 3} A 3 \\
Y=D_{Y F} F+D_{Y A 1} A 1+D_{Y A 2} A 2+D_{Y A 3} A 3
\end{array}\right.
$$

In Eq. (7), matrixes $D_{T T}, D_{T A 1}, D_{T A 2}, D_{T A 3}$ represent the relationship between the temperatures and the flame center migration with the fuels, primary airs, secondary airs and tertiary airs, respectively. The differences among the structures of matrixes $D_{X F}, D_{X A 1}, D_{X A 2}, D_{X A 3}$ and matrixes $D_{Y F}, D_{Y A 1}, D_{Y A 2}, D_{Y A 3}$ represent that fuels, primary airs, secondary airs and tertiary air have different influences on the flame centers. The depiction does accord with the practical operation conditions of boilers. The matrix $D$ in the models in Eqs. (3) and (7) can be determined by combustion simulation code, or by the analysis of the visualization of 3-D temperature distributions with the combustion conditions, and will not be described in detail here.

\section{OPTIMAL COMBUSTION CONTROL STRATEGY BASED ON GA}

Controlling the combustion processes in boilers with multi-burner configuration using the 3-D temperature distribution information inside the furnace as intermediate controlled parameters is an optimization problem with multi-parameter in which multiple inputs influence the multiple outputs. For such a problem to search for the optimal solution in a comprehensive and large search space, the prior knowledge about the problem must be utilized to lessen the search space and adaptively control the search process. GA is such an effective algorithm, which is initially put forward by professor John $\mathrm{H}$. Holland of Michigan University, is based on the Darwinian theory [10]. Through simulating the adaptive evolution process of biological systems, the GA presents its characteristics of mass search strategy and information exchange among individuals in the colony. Compared with conventional algorithms, GA does not directly operate on the variables or rely on the slope information of the problem. It utilizes a certain coding technique of variables, usually binary system coding, and is an algorithm that can be easily realized so long as the fitness information being known. Such a feature makes GA effectively solve discontinuous, 
irregular and nonconvex problems. Thereby, GA is applied widely in many fields, and is attracting increasing attention with academic scientists.

GA completely discards the mode of one-by-one solution. Its "colony effect" gives it a characteristic of searching a group of solution simultaneously, which makes GA more fit for the multi-objective optimal control systems. It is particularly useful for addressing goal-oriented multi-parameter optimization problems.

As far as optimal combustion control of the three-dimensional temperature distribution inside a furnace is concerned, GA is actually a linear optimal method, and fitness is the evaluation of solutions. At first, the input parameters of above model in Eqs. $(3,7)$ are discretized and coded with binary system. For the fixed, total fuel flowrate entering the furnace, the changes of the fuel flowrates of each burner is not independent. A way to gain the independence among the variables in $\mathrm{GA}$ is changing the absolute value of variables to relative ones of total amounts, where the chromosomes represent the ratio of variable values of each burner to total value of all burners. Letting that the value of certain chromosome be $F_{i}$, its practical value is given by $f_{i}=F_{i} / \sum F_{i}$. So, when the total value entering furnace keeps $1.0, F_{i}$ can be freely evaluated, and the independent operation on each variable in control strategy is realized. In practical GA based control program, the binary system coding the fuel value of each burner is 6-bit, and the primary and secondary airs are respectively 4-bit. They together consist the chromosome code of one burner. The binary system code of tertiary air is 4-bit, which is an independent chromosome. A data structure including chromosomes of all burners and that of tertiary air is an individual of GA. Evaluating above structure repeatedly with stochastic method can create a random-distributing initial colony with a scale of 100 .

The differences between the measured and optimal values of temperatures and migration of flame centers are used to form the fitness function, $V$, for the GA method, which is given as follows,

$$
V=0.009 \sum \sqrt[4]{\left|T_{i}^{4}-T_{0 i}^{4}\right|}+0.23 \sum \sqrt{\left(x_{i}-x_{0 i}\right)^{2}+\left(y_{i}-y_{0 i}\right)^{2}},
$$

where, $T_{0 i}, \quad\left(x_{0 i}, y_{0 i}\right)$ are the vectors of optimized temperatures and the coordinates of the flame centers in the several planes, respectively. Factors 0.009 and 0.023 in the function are the weights to balance the different magnitudes of temperatures and coordinates. In the expression, it can be seen that the weights for all the temperature differences are equal, so are those for the coordinates of the flame centers. The function of fitness is written as

$$
F_{F}=\left\{\begin{array}{lcc}
0 & \text { when } & V \geq 100 \\
100-V & \text { when } & 0 \leq V \leq 100
\end{array} .\right.
$$

Other practical processing steps, such as operator choice, crossover, variation and stop condition setting, are suggested to refer to the reference and choose the usual processing methods [8].

\section{SIMULATION AND ANALYSIS}

The control object is the tangential combustion process in the furnace of a boiler with 24 burners. The height of the furnace is $56.99 \mathrm{~m}$. The flame-diverting corner is located at the elevation of $44.15 \mathrm{~m}$. The section of furnace is $19.558 \mathrm{~m}$ wide and $16.432 \mathrm{~m}$ long. The burners, which are 6 layers, are mounted in the four corners of the furnace. The total height of the burners is $11.266 \mathrm{~m}$. The 3-dimensional combustion temperature distribution in the furnace is discretized with 10 planes along the height direction.

When this control strategy is applied in practice, the real-time temperature condition of all sections, $S$, is compared with the corresponding ideal temperature distribution $S_{0}$. When the difference exceeds the tolerance for the boiler, the control program begins to run. It diagnoses the cause of the difference, optimally adjusts the input parameters and then brings an optimized temperature distribution. The model used in the control program is,

$$
\Delta S=D \Delta U
$$

The resulted input parameter vector $\Delta U$ obtained using the GA method will lead to create a change in temperature distribution $\Delta S$ in furnace which just makes the temperature distribution back to its ideal condition.

By using the algorithm mentioned above, the control strategy is expected to search in the whole search space and converge speedily towards an optimal solution that meets the precision demand. To validate the control strategy, the algorithm program is tested under many kinds of disturbances of boiler combustion conditions. Following are the detailed optimized results and the analysis.

\section{1 Tracing Features of the Algorithm}

Firstly, the search process of GA for control variables during the course of its operation is tracesd. Results show that GA based control program can find the optimized solution from operators that distribute in the whole search space of the input parameters. Final individuals converge towards a global optimal solution. The fitness of individuals increases speedily to a degree that meets the precision demand. Fig. 2 and 3 give the search process of the control program. It can be seen that the solution found by GA based control strategy is a global optimal one. The search process is speedy, highly precious, and does avoid the deficiency of local optimal solution of conventional algorithms.

\subsection{Optimization Under Disturbance of Fuel Heating Value}

When the quality and the heating value of fuel change, if the total fuel flowrate into the furnace keeps fixed and the ratios of the flowrates of the primary and secondary airs to that of the fuel of each burner are unaltered, the total heat entering the furnace will change, and accordingly the temperatures and the flame centers in each plane will 


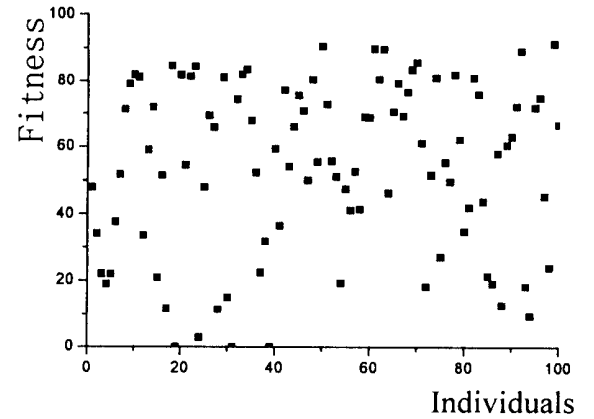

Figure 2. Distribution of fitness of initial colony

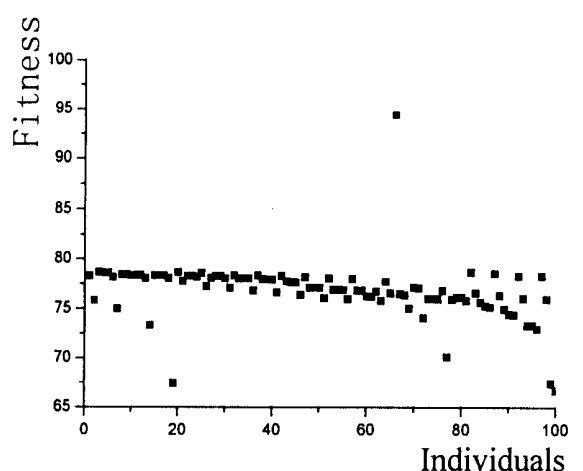

Figure 3. Distribution of fitness of optimized colony

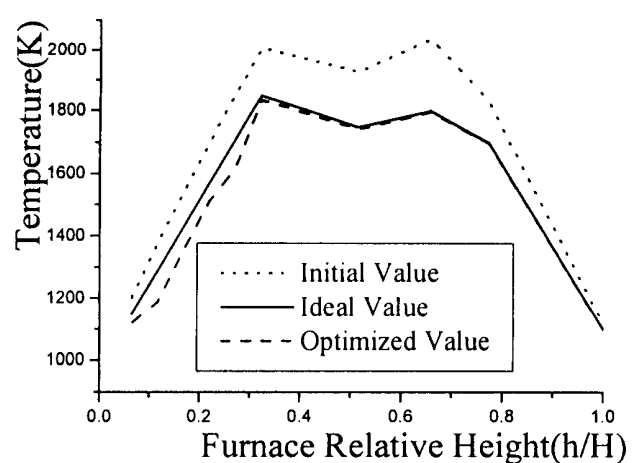

Figure 4. Optimized temperature when heating value increases

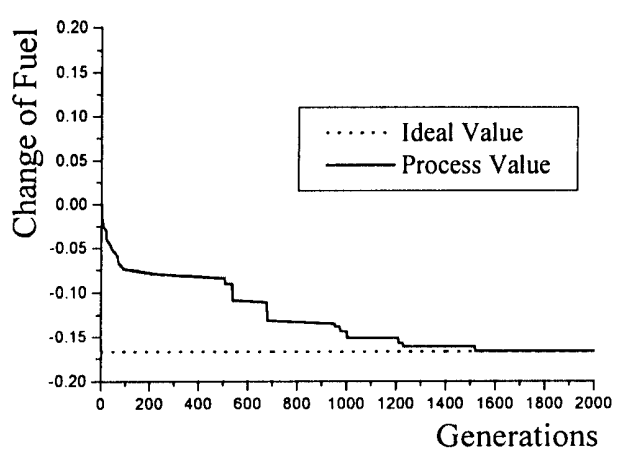

Figure 5 Change of the fuel flowrate

change too. As the relative momentum in each burner is unaltered, the horizontal positions of flame centers will keep unchanged. Fig. 4 shows the temperature distribution before and after optimization by the control strategy when the fuel heating value changes suddenly to 1.2 times. Results demonstrate that the control strategy can speedily diagnose the disturbance of the combustion system and adjust it. From Fig. 4, it can also be seen that except in the bottom of the furnace, there is only a little difference between the optimized temperature and the prescribed ideal one. Fig. 5 also reveals the optimization of total fuel input, which decreases gradually from the bigger value under the disturbance condition to the optimal value during the course of the searching process. The similar results are obtained when the fuel heating value decreases suddenly to 0.8 times as the previous.

It is noted that the control process has little influence on the horizontal positions of flame centers during the course of the temperature value approaching to the optimal state. Whether the heating value increases or decreases, the $x$ - and $y$-axis biases of flame centers are not sensitive.

\subsection{Optimized Results Under Disturbance of Load}

Any boiler in power generation stations should operate under different load levels, which will be ensured by the change of the 3-D distributions of temperatures and radiative properties in the flame in furnace. When the load changes, the flowrates of fuel and air fed into the furnace should be adjusted, and then the temperatures and the radiant emissivity of the flame will change too. According to the Planck's law of radiation, the total radiative energy transferred to the waterwall surface of the boiler can be figured out from the temperatures and the radiant emissivity of the flame. When the load requirement for the unit increases 1.2 times, Fig. 6 shows the optimized results of temperatures of the flame. Fig. 7 and 8 show the optimized results of the flowrates of the fuels, primary and secondary airs of each burner. Each burner will receive an instruction of increasing the fuel flowrate, and the total fuel flowrate entering the furnace increases to about 1.2 times as before. The primary and secondary air flowrates of each burner are increased accordingly. Results reveal that the GA based control strategy can rapidly respond to the requirement of changing boiler load and adjust the fuel and air flowrates of each burner. The adjustment makes the temperature distribution close to the ideal temperature distribution under the new boiler load level. Simultaneously, the horizontal $x$ - and $y$-axis biases of the flame centers are controlled well. The similar optimization effect could be seen when the boiler load decreases to 0.8 times.

\subsection{Optimization Results Under Disturbance of Burner Failure}

When a burner is tripped and its fuel flowrate decreases suddenly, or when there is a trouble with air feeding devices, the total fuel flowrate and the fuel-to-air ratio will depart from their ideal values, which will change the combustion state in the furnace. Under such conditions, the 3-D temperature distribution of the flame will depart from the ideal one, in which not only the temperatures in the whole furnace will change, but also the coordinates of flame centers will also alter from the geometric centers in different heights. The control strategy introduced by this paper can diagnose in real-time and optimize the combustion state rapidly. Fig. 9 to 12 


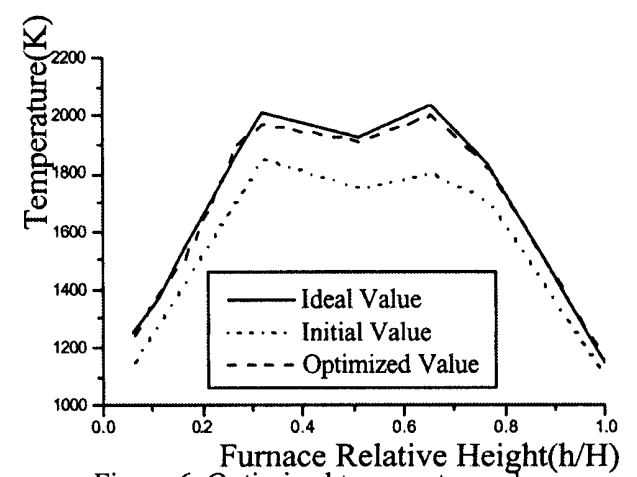

Figure 6. Optimized temperature when

load increases

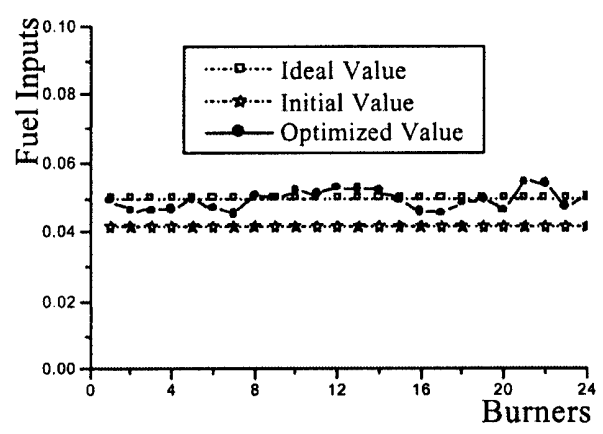

Figure 7. Optimized fuel flowrates when load increases

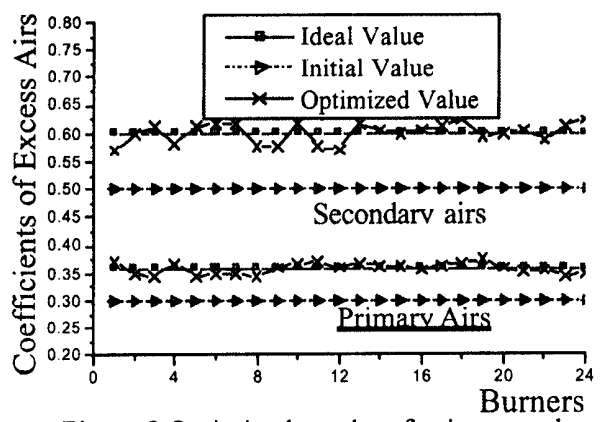

Figure 8.Optimized results of primary and scondary air flowrates

represent the optimized results of temperature distribution compared with the initial distribution when a right-hand-side burner in the third layer fails. If the controller is given an instruction that this burner cannot be resumed, the algorithm program then fixes the fuel flowrate of that burner to zero, and during the course of optimization, the fuel flowrates of several burners near the failed one increase (as shown in Fig. 9), and the primary and second air flowrates are optimized correspondingly. This adjustment can compensate the influence on the temperatures and the biases of flame centers due to the failure of the burner. If it is given that the burner can be resumed, the optimize result is that the fuel and the air flowrates of the burner will be forced back to the normal ones (as shown in Fig. 9), and the combustion state doesn't change obviously. So, the results have revealed that the control strategy can rapidly diagnose the disturbance of the burner failure and optimize the combustion state in the

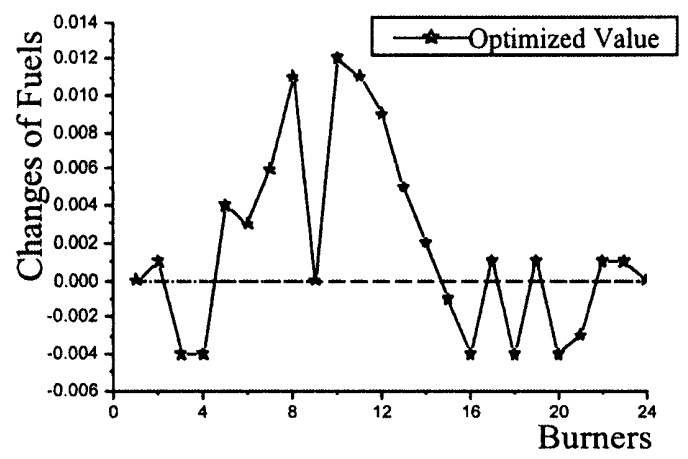

Figure 9. Optimized fuel flowrates when one burner fails

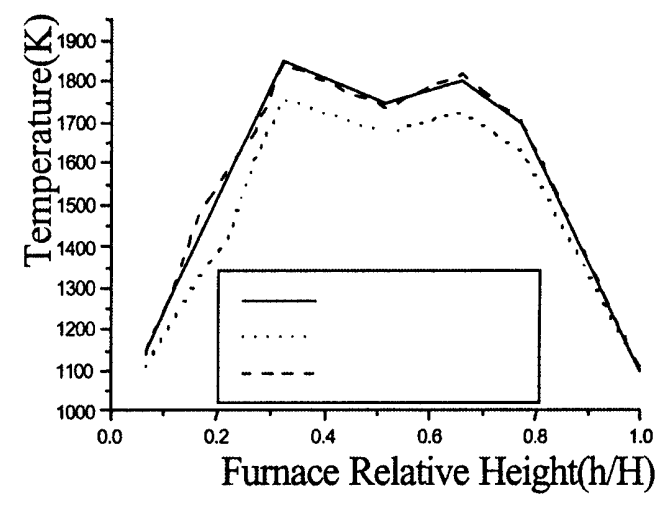

Figure 10. Optimized temperatures when one burner fails

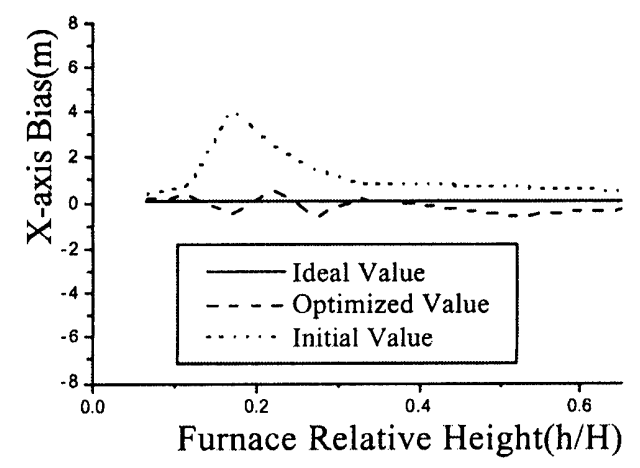

Figure 11. $\mathrm{X}$-axis bias when one burner fails

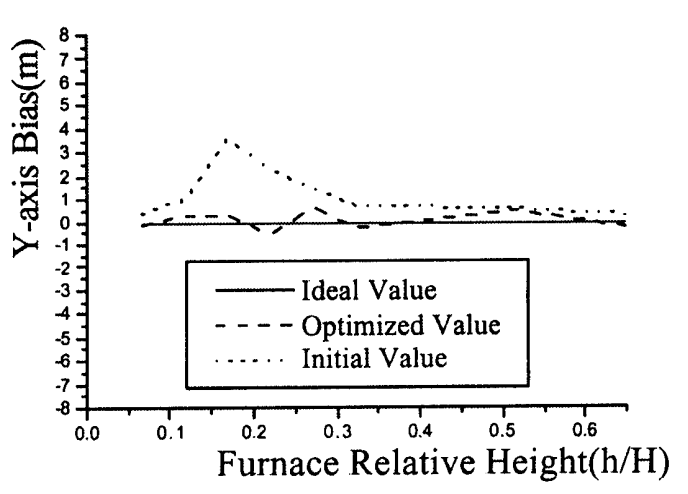

Fig. $12 \mathrm{Y}$-axis bias when one burner fails 
furnace.

\section{CONCLUSIONS}

The genetic algorithm is introduced into an optimal control method for pulverized coal combustion in multi-burner furnaces. The three-dimensional temperature distribution is used as a set of intermediate variables, and its optimization is taken as a basic aim. The simulation has been done with a furnace combustion model, and the results show that the control method can overcome the impact of the disturbance of the heat value of fuel on the combustion process, provide suitable control signals for the fuel and air flowrates to change the load level, and estimate the failure of individual burner and give correct control signals to deal with it. At present, we are developing a system to visualize the 3-D combustion temperature distributions in furnaces. The work is expected to establish a firm basis for the application of the optimal control strategy introduced by the paper.

\section{AKNOWLEDGEMENTS}

The present study has been supported by the National Natural Science Foundation of China (No. 50176056), and the Teaching and Research Award Program for Outstanding Young Teachers in Higher Institutions of MOE, P. R. China.

\section{REFERENCES}

1. Kocijan, J., An Approach to Multivariable Combustion Control Design, Journal of Process Control, Vol.7 (1997), pp.291-301.

2. John, D. J. and Samuelsen, S., Robust Optimal Control of a Natural Gas-Fired Burner for the Control of Oxides of Nitrogen (NO), Combustion Science and Technology, Vol. 128 (1997), pp.1-21.

3. Liu, G. S., and Daley, S., Design and Implementation of an Adaptive Predictive Controller for Combustor NOx Emissions, Journal of Process Control, Vol.9 (1999), pp.485-491.
4. Ptscheider, K. and Welfonder, E., Modelbased Online Minimization of NOx-emission in Power plants with Pulverized Coal Combustion, Proceedings of the 13th World Congress, Volume O, Power Plants and Systems, Computer Control, Edited by J. J. Gertler, J. B. Cruz Jr and M. Peshkin, (1996), pp.49-54.

5. Fujii, S., Tomiyama, S., Nogami, Y., Shirai, M., Ase, H. and Takashi Yokoyama, Fuzzy Combustion Control for Reducing Both $\mathrm{CO}$ and NOx from Flue Gas of Refuse Incineration Furnace, JSME International Journal, Series C, Vol.40 (1997), pp.279-284.IFAC, San Francisco, USA.

6. Zhou, H. C., Sheng F., et al., Reconstruction of Temperature Distribution in a 2-D Absorbing-Emitting System from Radiant Images, JSME International Journal, Series B, Vol.43 (2000), pp.104-109.

7. Wang F., Ma Z. Y., Wei C. Y., Yan J. H., Cen K. F., Study on Section Temperature Measurement of Power Plant Boiler Based on Flame Images, Proc Chinese Soc Elect Eng, Vol.20(7) (2000), pp40-44.

8. Zhou H. C., Han S. D., Sheng F., Zheng C. G., Visualization of Three-Dimensional Temperature Distributions in a Large-Scale Furnace via Regularized Reconstruction from Radiative Energy Images: Numerical Studies. JQSRT, Vol.72(4) (2002), pp.361-383.

9. Obertacke, R., Wintrich, H., Wintrich F., and Leipertz, A., A New Sensor System for Industrial Combustion Monitoring and Control Using UV Emission Spectroscopy and Tomography, Combustion Science and Technology, Vol.121 (1996), pp. 133-151.

10. Vavak, F., SHARPE, P. K., Balancing Fuel-to-Air Ratio In Multiple Burner Boiler Using Genetic Algorithm Based Control System . CLEAN AIR IV, (1997), pp.14.1., Calouste Gulbenkian Foundation Lisbon-Portugal

11. Chen G. L., Wang X. F., Genetic Algorithm and Its Applications, (1996), The Public's Posts and Telecommunications Press, Beijing. 